\title{
Raised dopamine metabolites in a case of malignant paraganglioma
}

\author{
C.M. Florkowski', D.J. Fairlamb², M.G. Freeth ${ }^{3}$, S.A. Taylor ${ }^{4}$, A. Taylor ${ }^{5}$, \\ C. Weinkove ${ }^{5}$ and A.G. Jacobs ${ }^{1}$
}

\begin{abstract}
Departments of ${ }^{1}$ Clinical Chemistry, ${ }^{2}$ Oncology, ${ }^{3}$ Pathology, and ${ }^{4}$ Surgery, New Cross Hospital, Wolverhampton WV10 0QP and ${ }^{5}$ Department of Clinical Biochemistry, Hope Hospital, Salford and University of Manchester, $U K$.
\end{abstract}

\begin{abstract}
Summary: This paper describes the case of a malignant retroperitoneal paraganglioma with extensive metastases. The patient presented with a supraclavicular mass and an absence of hypertension. Exclusively raised dopamine metabolites were detected which may be a marker of a malignant process and account for the lack of hypertension.
\end{abstract}

\section{Introduction}

Catecholamine producing tumours may arise within the adrenal medulla or at sites of paraganglionic tissue including the carotid body, glomus jugulare, vagus nerve, mediastinum, retroperitoneum and bladder. ${ }^{1}$ There are no consistently reliable histological or biochemical features to distinguish benign from malignant tumours of this type. ${ }^{2}$ The presence of raised dopamine metabolites may, however, be a marker of malignancy as has been described for adrenal phaeochromocytoma ${ }^{3}$ and in a previous case of paraganglioma. ${ }^{4}$ The present case illustrates the occurrence of exclusively raised dopamine metabolites in a case of malignant retroperitoneal paraganglioma with a notable absence of hypertension.

\section{Case report}

A 26 year old male presented with a mass in the left supraclavicular fossa and increasing dyspnoea. He had been unwell for a period of 18 months although he had not previously sought medical advice. On examination he was frail, anaemic and dyspnoeic with a large left sided neck mass and bilateral pleural effusions. Blood pressure was $150 / 60 \mathrm{mmHg}$ and it is noteworthy that he remained consistently normotensive throughout the course of his illness. There was hepatomegaly and an irregular $10 \mathrm{~cm}$ central abdominal mass. The initial clinical impression was of a disseminated lymphoreticular malignancy although no other lymph node groups were enlarged.

Correspondence: C.M. Florkowski, M.A., M.R.C.P. Accepted: 3 January 1990
Chest X-ray confirmed bilateral pleural effusions. A total of 2 litres of exudate was aspirated from the left pleural cavity and found to contain lymphocytes and reactive mesothelial cells but no malignant cells. Abdominal ultrasound revealed a huge mass of tissue, probably para-aortic nodes, extending to the left and surrounding a distorted, hydronephrotic left kidney. A computerized tomographic (CT) scan of the abdomen demonstrated a large mass of lymph nodes extending from the retrocrural area with a maximum diameter of $15-20 \mathrm{~cm}$ involving the lesser omentum, surrounding the pancreatic, para-aortic and paracaval areas and extending to the pelvic brim. There were also deposits in both the right and left lobes of the liver and evidence of bilateral common iliac lymphadenopathy.

Haematological investigations showed a haemoglobin of $9.6 \mathrm{~g} / \mathrm{dl}$, a normochromic normocytic blood film and an ESR of 31. Urea and electrolytes were normal. The alkaline phosphatase was raised at 2,240 IU/1 (reference range 100-350) and also alanine transaminase at $113 \mathrm{IU} / 1$ (reference range 5-40) but with a normal bilirubin.

Surgical biopsy of the neck mass was performed. Histological examination showed a well defined nodule of tumour tissue with a pronounced alveolar pattern, interspersed with fibrosepta. Nuclear pleomorphism was mild although mitotic figures were found. Invasion of the tumour into small vascular spaces was seen at the periphery of the lesion. The chromaffin reaction of fresh tissue was negative although the argyrophil reaction was positive. Immunoperoxidase stains for neuronespecific enolase (NSE) and S100 protein showed strong staining for NSE and weaker staining for S100. Histological findings were therefore consis- 
tent with a malignant paraganglioma, most probably a metastasis from a retroperitoneal tumour in view of the lack of relationship of the neck mass to the carotid body.

Thin layer chromatography of urine for catecholamine metabolites demonstrated a strongly staining band for homovanillic acid (HVA), a metabolite of dopamine, with no evidence of raised vanillylmandelic acid (VMA). Urinary VMA and HVA levels were determined by capillary gas-liquid chromatography (GLC) on a Perkin Elmer 8600 chromatograph with flame ionization detection following extraction into ethyl acetate and derivatization with $N$-methyl- $N$-trimethylsilylfluoroacetamide (MSTFA), using tropic acid as an internal standard. ${ }^{5}$ HVA was raised at $138.7 \mathrm{mg} / \mathrm{g}$ creatinine (reference range up to 15.6$)^{5}$ while VMA was not raised at $2.0 \mathrm{mg} / \mathrm{g}$ (reference range up to 7.8 ). ${ }^{5}$ GLC therefore demonstrated exclusively raised dopamine metabolites.

The patient received a single course of chemotherapy with ifosfamide $5 \mathrm{~g} / \mathrm{m}^{2}$ over 24 hours and mesna $7 \mathrm{~g} / \mathrm{m}^{2}$ over 36 hours. He showed an initial clinical improvement although subsequently deteriorated and died approximately one month after presentation.

Post-mortem examination demonstrated a large mass of tumour occupying the peritoneum roughly between the kidneys and extending over the superior aspect of the left kidney. The tumour was approximately $20 \mathrm{~cm}$ in diameter composed of multiple, small, fairly well defined nodules.

It completely encased the aorta and extended down to the brim of the pelvis and along the common iliac artery. The left kidney was pale and hydronephrotic due to ureteric obstruction by the mass of retroperitoneal tumour. Nodules of tumour including one of $3 \mathrm{~cm}$ diameter were present in the liver. The right adrenal gland was normal although the left adrenal could not be found. It was impossible to determine whether the tumour had arisen within the left adrenal or had invaded and replaced it. It was considered more likely to be a primary retroperitoneal tumour in view of the fact that the main mass of tumour was central surrounding the aorta.

Catecholamine levels were determined in samples of tumour tissue by HPLC following extraction into hydrochloric acid $(1 \mathrm{~mol} / \mathrm{l}){ }^{6}{ }^{6}$ The content of noradrenaline was found to be $5.9 \mathrm{nmol} / \mathrm{g}$ wet weight compared with a reference range of $240-960 \mathrm{nmol} / \mathrm{g}$ for normal adrenal tissue. ${ }^{7}$ Adrenaline was found to be $<2.3 \mathrm{nmol} / \mathrm{g}$ wet weight (reference range $1200-4600 \mathrm{nmol} / \mathrm{g}$ ). ${ }^{8}$ The dopamine level was $577 \mathrm{nmol} / \mathrm{g}$ wet weight which is above a reference range of $41-274 \mathrm{nmol} / \mathrm{g}$ found in a series of patients with neuroblastoma, a tumour that predominantly synthesizes dopamine. ${ }^{8}$

The final diagnosis was of a malignant retro- peritoneal paraganglioma with extensive metastases and exclusively raised dopamine metabolites.

\section{Discussion}

The classification of extra-adrenal paraganglioma according to Glenner and Grimley is based on anatomical location, histochemical features and innervation. ${ }^{1}$ The four categories include branchiomeric, intravagal, aortico-sympathetic and visceroautonomic paraganglioma. The present case falls within the category of aortico-sympathetic although this is synonymous with retroperitoneal and also organ of Zuckerkandl paraganglioma. The term phaeochromocytoma should strictly be limited to tumours arising within the adrenal medulla. Both chromaffin ${ }^{9}$ and non-chromaffin ${ }^{10}$ functional tumours of the retroperitoneal paraganglioma have been described but there is no direct correlation of the staining characteristics with the activity of the tumour. The tumour in the present case exhibited a negative chromaffin reaction.

An association between malignant phaeochromocytoma and dopamine was suspected by McMillan in 1956. ${ }^{11}$ Feldman et al. were the first to establish a relationship between the absence of hypertension and the preferential excretion of dopamine and that, in such a tumour, decreased activity of the enzyme dopamine beta hydroxylase suggests poor differentiation. ${ }^{12}$ In a series of 50 patients with phaeochromocytoma Proye et al. showed exclusively raised dopamine levels in 3 cases that were normotensive. ${ }^{13}$

It was thought that the predominant or exclusive secretion of dopamine would explain the lack of hypertension due to its anti-adrenergic action that inhibits the vasoconstrictive effects of other amines. Furthermore in the same series of patients, of those that secreted dopamine (whether exclusively or together with other catecholamines) $66 \%$ were malignant compared with $30.8 \%$ of those that did not secrete dopamine. In another series of 14 patients with malignant phaeochromocytoma, Tippett $e t$ al. found increased dopamine excretion in $9(64 \%)$ subjects including all 4 with primary intra-adrenal tumours and that this was associated with a poor prognosis. ${ }^{3}$ This is a higher proportion than in other similar studies ${ }^{14}$ and may be due to better assessment of metastatic spread and thus the definition of malignancy by radio-pharmaceutical imaging with ${ }^{131}$ I-meta-iodobenzylguanidine. A case of malignant chemodectoma (branchiomeric paraganglioma) has also been described with elevated urinary HVA in a patient that did not have raised blood pressure. ${ }^{4}$ Raised levels of dopamine and its metabolites may therefore be a better marker of malignancy than hitherto suspected.

The presence of raised dopamine (and its meta- 
bolites) may therefore reflect the lack of differentiation of tumour tissue and be a marker of malignancy as suggested in the present case. The absence of hypertension presents a further diagnostic

\section{References}

1. Glenner, G.G. \& Grimley, P.M. Tumours of the extraadrenal paraganglion system (including chemoreceptors). In: Atlas of Tumour Pathology, second series, fascicle 9. Armed Forces Institute of Pathology, Washington DC, 1974, pp. 14-77.

2. Ledingham, J.G.G. In: Weatherall, D.J., Ledingham, J.G.G. \& Warrell, D.A. (eds) Oxford Textbook of Medicine, 2nd Edition. Oxford Medical Publications, Oxford, 1987, p. 13.393.

3. Tippett, P.A., McEwan, A.J. \& Ackery, D.M. A re-evaluation of dopamine excretion in phaeochromocytoma. Clin Endocrinol 1986, 25: 401-410.

4. Enquist, R.W., Tormey, D.C., Jenis, E.H. \& Warkel, R.L. Malignant chemodectoma of the superior mediastinum with elevated urinary homovanillic acid. Chest 1974, 66: 209-211.

5. Price, S., Davenport, K. \& Berg, J.D. Determination of urinary 3-methoxy-4-hydroxymandelic and 3-methoxy-4hydroxyphenylacetic acids by fused silica capillary gas chromatography. Ann Clin Biochem 1986, 23: 351-353.

6. Weinkove, C., Adrenaline, noradrenaline and related compounds. In: Gowenlock, A.H., McMurray, J.R., McLauchlan, D.M. (eds) Varley's Practical Clinical Biochemistry, 6th Edition. Heineman Medical Books, London, 1988 , pp. 883-885.

7. Von Euler, U.S. Epinephrine and norepinephrine. Adrenaline and noradrenaline. Distribution and action. Pharmacol Rev 1954, 6: 15-22. challenge and it is important to consider this possible diagnosis in the presence of unexplained masses and to selectively test the urine for dopamine or its metabolite HVA.

8. Gjessing, L.R. Studies of functional neural tumours. VI. Biochemical diagnosis. Scand J Clin Lab Invest 1964, 16: $661-669$.

9. Isaacson, C., Rosenzweig, D. \& Seftel, H.C. Malignant pheochromocytoma of the organs of Zuckerkandl. Arch Pathol 1960, 70: 725-729.

10. Attia, A., Golden, R.L. \& Ziffer, H. Nonchromaffin staining functional tumour of the organs of Zuckerkandl. $N$ Engl $J$ Med 1961, 264: 1130-1134.

11. McMillan, C. Identification of hydroxytyramine in a chromaffin tumour. Lancet 1956, ii: 284.

12. Feldmann, J.M., Blalock, J.A., Zern, R.T. et al. Deficiency of dopamine-beta-hydroxylase. A new mechanism for normotensive phaeochromocytomas. Am J Clin Pathol 1979, 72: $175-185$.

13. Proye, C., Fossati, P., Fontaine, P. et al. Dopamine secreting phaeochromocytoma: an unrecognized entity? Classification of pheochromocytomas according to their type of secretion. Surgery 1986, 100: 1154-1161.

14. Serrano, P.A., Chavez-Lara, B. \& Sanchez-Torres, G. Dopamine excretion in benign pheochromocytoma. Biomedicine 1978, 29: 229-231. 